\title{
Relapsed Plasmablastic Lymphoma in a HIV negative patient: pushing the envelope
}

\author{
Manthan Kathrotiya ${ }^{1}$, Vivek Radhakrishnan ${ }^{1}$, Saurabh Bhave ${ }^{1}$, Jeevan Kumar ${ }^{1}$, Mita \\ Roychowdhury ${ }^{1}$, Indu Arun ${ }^{1}$, Jayanta Das $^{1}$, Mammen Chandy ${ }^{1}$, and Reena Nair ${ }^{1}$ \\ ${ }^{1}$ Tata Medical Center
}

August 4, 2020

\begin{abstract}
We report a case of Plasmablastic Lymphoma occurring in a 45-year-old HIV-negative woman involving extra nodal sites including ovary, breast and adrenal glands. Prognostic outcomes are generally dismal. On relapse, she received conventional as well as novel therapies and is disease free now, two years post allogenic haploidentical transplantation.
\end{abstract}

Keywords:

PLASMABLASTIC LYMPHOMA, RELAPSE, NOVEL THERAPIES, DARATUMUMAB, HAPLOIDENTICAL TRANSPLANTATION

Key Clinical Message:

This case emphasises that, with the availability of novel agents (Daratumumab), and repurposed use of bortezomib, even relapsed PBL could be treated successfully and consolidated with an allogeneic haploidentical hematopoietic cell transplantation.

\section{Introduction:}

Plasmablastic lymphoma (PBL) is a rare and aggressive CD20-negative B-cell Non-Hodgkin Lymphoma (B-NHL) associated with modest outcomes in HIV positive, and poor outcomes in the HIV Negative. HIVnegative PBL patients are known to present with an advanced clinical stage (less common bone marrow involvement), B symptoms, and have significantly poorer outcomes when compared to HIV-positive patients (1). Multiple studies have shown median survival times of $12-18$ months. $(1,2)$

\section{Case Presentation:}

A 45-year-old Chinese Indian woman presented in Feb 2015 with complaints of 'persistent' back pain and a recent history of total hysterectomy for an ovarian mass diagnosed as ovarian plasmacytoid neoplasm. She had no other localizing symptoms, no B symptoms and informed us of a family history of breast cancer (mother). Examination revealed bilateral (B/L) breast lumps (3cms max). CT thorax and abdomen showed additional lesions including few small left axillary lymph nodes, nodular lesions in bilateral adrenal glands $(4.2 \mathrm{~cm} \max )$ and altered signals in L1 vertebrae with an associated paravertebral soft tissue mass $(5.3 \mathrm{~cm}$ max) compressing the conus. HIV test (by ELISA) was negative. Biopsy of the breast lumps showed lymphoproliferative neoplasm with plasmacytoid histology [positive for CD138, EBER-ISH, and kappa light chain restriction] s/o Plasmablastic Lymphoma. Bone marrow (BM) aspirate showed $6 \%$ plasma cell with no atypical cells. Cytogenetics on Marrow was FISH 17p deleted in $8 \%$ plasma cells. Baseline Protein electrophoresis was not done. She received local RT for pain control in the dorso-lumbar region. With a curative intent, she was treated with 6 cycles of dose-adjusted EPOCH (daEPOCH). After 6 cycles of 
daEPOCH PET CT showed complete metabolic response (cMR). This was consolidated with BEAM high dose chemotherapy and autologous hematopoietic stem cell transplant (AHCT) in Aug 2015. Follow up PET-CT 3 months after AHCT continued to be in cMR. On regular follow-up, in Jan 2017, she relapsed with B/L breast lumps (6cms max) \soutand confirmed by repeat biopsy (CD138 and CD 38 positive). PET $\mathrm{CT}$ showed an additional lytic lesion in the seventh rib. BM and CSF were uninvolved. cMYC rearrangement was negative. She received salvage DHAP chemotherapy from Feb 2017. PET-CT post 3 cycles, showed reduction in size of breast lumps but progression with new lesions in ribs. She was switched to a Bortezomib and Lenalidomide (VR) regimen from May 2017. Daratumumab(D) was added from July 2017. After 4 cycles of combination DaraVR, PET-CT showed mild residual metabolic activity in bilateral breast lesions. She received local radiotherapy (RT) to B/L breast (45 Gy in 25\# over 5 weeks). Post RT, PET-CT showed cMR. This was followed by a haploidentical allogenic hematopoietic cell transplant (AlloHCT) using T-cell replete peripheral blood stem cells from her haploidentical sibling brother in Dec 2017. Conditioning regimen used was myeloablative Fludarabine-Busulfan. Graft versus host disease (GVHD) prophylaxis consisted of a combination of post-transplant cyclophosphamide, tacrolimus and mycophenolate (PTCy+Tac+MMF). Peri-transplant period was uneventful. She had complete engraftment by Day +15 . In April 2018, by Day +94 , she developed Skin and Gut GVHD for which she received Methylprednisolone $1 \mathrm{mg} / \mathrm{kg}$ along with oral budesonide, and Tacrolimus (oral) was continued. GVHD settled thereafter. She had a relapse of skin GVHD on Day +189 which settled with steroid re-challenge. Her immunosuppression was later tapered and stopped by 12 months post-transplant. A PET CT repeated in Oct 2019 showed cMR. At last follow up in March 2020, she remained disease free after more than 2 years of AlloHCT.

\section{Discussion:}

PBL is a rare type of high-grade B-NHL, frequently involving extra nodal sites(1). Our patient had multiple extra-nodal site sites of involvement at presentation (ovary, breast, adrenal gland, vertebra and para-vertebral soft tissue) An important differential diagnosis for PBL is plasmablastic myeloma. Absence of bone marrow involvement with abnormal plasma cells, or myeloma related end-organ dysfunction makes plasmablastic myeloma an unlikely diagnosis (3). We reviewed literature and found that CHOP (cyclophosphamide, doxorubicin, vincristine, and prednisone) or CHOP-like regimens like Hyper-CVAD-MA (hyperfractionated cyclophosphamide, vincristine, doxorubicin, dexamethasone, and high-dose methotrexate and cytarabine), CODOX-M/ IVAC (cyclophosphamide, vincristine, doxorubicin, high-dose methotrexate/ ifosfamide, etoposide, and high-dose cytarabine), COMB (cyclophosphamide, oncovin, methyl-CCNU, and bleomycin), and infusional EPOCH (etoposide, prednisone, vincristine, cyclophosphamide, and doxorubicin) have been used (4). Polychemotherapy achieves more than $50 \%$ complete remissions (CRs) in patients with disseminated disease (2). Though the role of infusional EPOCH has not been proven effectively, its use is supported by the impressive results achieved in HIV-associated Non-Hodgkin's Lymphoma. This has become the treatment of choice in many institutions (5). Short series have been reported on the role of AHCT in both HIV and immunocompetent patients. A multi-center Phase II GIGAT group study included 5 patients with PBL, among other lymphoma types, who underwent an AHCT after first CR. Four of them maintained CR after 13-83 months (6) . Considering the aggressive course, PBL patients with high-risk features should be considered for consolidation with AHCT in first-line setting (7). Our patient received daEPOCH chemotherapy and was consolidated with AHCT in CR1. She remained in remission for 1 year and 4 months, which is consistent with the median overall survival (OS) reported elsewhere (8). Factors affecting survival in PBL are the following: achieving complete remission (CR), performance status, clinical stage, MYC gene rearrangements, and International Prognostic Index (9) . Our patient relapsed in the breast in Jan 2017 and had insignificant response to 3 cycles of salvage chemotherapy (DHAP). She was started on bortezomib and lenalidomide (VR). A literature review by Guerrero-Garcia TA et al, on the use of bortezomib along with chemo regimens in PBL patients observed higher response and survival rates in the frontline setting, and impressive transient responses in relapsed cases (10). Other agents like lenalidomide, brentuximab vedotin, and Interleukin 6 directed agents have been shown to benefit in PBL in a few case reports $(11,12)$. Few case reports of daratumumab, an antibody directed against CD38, have shown activity in patients with relapsed PBL (13). Daratumumab was added along with VR from cycle 3. After 4 cycles of combination DaraVR 
and Radiation therapy, PET CT was in cMR. There are very few cases of successful AlloHCT in PBL in HIV positive patients (14). She underwent an AlloHCT with haplo-identical sibling brother. She developed limited gut and skin GVHD which responded to steroids. She is alive now 4 years post diagnosis of PBL and more than 2 years post AlloHCT with no evidence of disease, and is off immunosuppression. The emergence of biology driven approaches like novel B-cell targets and immunotherapeutic approaches like cellular immunotherapy (CD30 directed CAR T-cell); anti-PD-1 or anti-PDL-1 monoclonal antibodies; EBV directed antiviral agents (arginine butyrate, ganciclovir), bromodomain extra terminal (BET) inhibitor for MYC gene positive cases; and PI3K/Akt/m-TOR pathway inhibitors hold promise (15). This case exemplifies the fact that when clinical compulsions push treating teams to explore options other than the conventionally tried methods in a hard to treat cancer, outcomes can be rewarding occasionally. This patient received both conventional and novel therapies, and was later consolidated with a haploidentical AlloHCT.

\section{Conclusion:}

1. This case emphasizes that with the availability of novel agents (Daratumumab) and use of bortezomib, even refractory PBL could be treated successfully.

2. This case signifies that daEPOCH followed by upfront autologous stem cell transplant in CR1 is a suitable option in primary PBL giving durable remission.

3. To the best of our knowledge this is the first reported case of a relapsed HIV negative PBL treated with AlloHCT who is disease-free for more than 2 years.

\section{References:}

1. Castillo JJ, Winer ES, Stachurski D, Perez K, Jabbour M, Milani C, et al. Clinical and pathological differences between human immunodeficiency virus-positive and human immunodeficiency virus-negative patients with plasmablastic lymphoma. Leuk Lymphoma. 2010;51(11):2047-53.

2. Tchernonog E, Faurie P, Coppo P, Monjanel H, Bonnet A, Algarte Génin M, et al. Clinical characteristics and prognostic factors of plasmablastic lymphoma patients: analysis of 135 patients from the LYSA group. Ann Oncol. 2017;28(4):843-8.

3. Morscio J, Dierickx D, Nijs J, Verhoef G, Bittoun E, Vanoeteren X, et al. Clinicopathologic comparison of plasmablastic lymphoma in HIV-positive, immunocompetent, and posttransplant patients: single-center series of 25 cases and meta-analysis of 277 reported cases. Am J Surg Pathol. 2014;38(7):875-86.

4. Zelenetz AD, Abramson JS, Advani RH, Andreadis CB, Byrd JC, Czuczman MS, et al. NCCN Clinical Practice Guidelines in Oncology: non-Hodgkin's lymphomas. J Natl Compr Canc Netw. 2010;8(3):288-334.

5. Sparano JA, Lee JY, Kaplan LD, Levine AM, Ramos JC, Ambinder RF, et al. Rituximab plus concurrent infusional EPOCH chemotherapy is highly effective in HIV-associated B-cell non-Hodgkin lymphoma. Blood. 2010;115(15):3008-16.

6. Re A, Michieli M, Casari S, Allione B, Cattaneo C, Rupolo M, et al. High-dose therapy and autologous peripheral blood stem cell transplantation as salvage treatment for AIDS-related lymphoma: long-term results of the Italian Cooperative Group on AIDS and Tumors (GICAT) study with analysis of prognostic factors. Blood. 2009;114(7):1306-13.

7. Al-Malki MM, Castillo JJ, Sloan JM, Re A. Hematopoietic cell transplantation for plasmablastic lymphoma: a review. Biol Blood Marrow Transplant. 2014;20(12):1877-84.

8. Qunaj L, Castillo JJ, Olszewski AJ. Survival of patients with CD20-negative variants of large B-cell lymphoma: an analysis of the National Cancer Data Base. Leuk Lymphoma. 2018;59(6):1375-83.

9. Schommers P, Wyen C, Hentrich M, Gillor D, Zoufaly A, Jensen B, et al. Poor outcome of HIV-infected patients with plasmablastic lymphoma: results from the German AIDS-related lymphoma cohort study. Aids. 2013;27(5):842-5. 
10. Castillo JJ, Guerrero-Garcia T, Baldini F, Tchernonog E, Cartron G, Ninkovic S, et al. Bortezomib plus EPOCH is effective as frontline treatment in patients with plasmablastic lymphoma. Br J Haematol. 2019;184(4):679-82.

11. Pretscher D, Kalisch A, Wilhelm M, Birkmann J. Refractory plasmablastic lymphoma-a review of treatment options beyond standard therapy. Ann Hematol. 2017;96(6):967-70.

12. Koenig G, Stevens TM, Peker D. Plasmablastic microlymphoma arising in human herpesvirus-8associated multicentric Castleman disease in a human immunodeficiency virus-seronegative patient with clinical response to anti-interleukin-6 therapy. Histopathology. 2015;67(6):930-2.

13. Chikeka I, Grossman M, Deng C, Jacob AT, Husain S. Plasmablastic lymphoma in an HIV patient with cutaneous presentation: A case of remarkable remission in a typically refractory disease. JAAD Case Rep. $2020 ; 6(3): 161-5$.

14. Hamadani M, Devine SM. Reduced-intensity conditioning allogeneic stem cell transplantation in HIV patients with hematologic malignancies: yes, we can. Blood. 2009;114(12):2564-6.

15. Lopez A, Abrisqueta P. Plasmablastic lymphoma: current perspectives. Blood Lymphat Cancer. 2018;8:63-70.

Author Contributions: VSR and RN: conceived and lead the idea for the case report; VSR, MK and RN wrote the manuscript, contributed to the design and editing the manuscript. IA and JD contributed pathology and imaging inputs (respectively), pictures and edited the manuscript. VSR, RN, MK, SB, MR, JK, and MC: examined and treated the patient, and edited the manuscript.

Figure Legends:

Figure 1. A) and B): Hematoxylin and Eosin stain shows tumour cells in sheets with rounded nuclei, coarse chromatin, single nucleoli and moderate cytoplasm, low power and high power images respectively.C) CD38 immunohistochemistry (IHC) stain

Figure 2: A to $\mathbf{F}$ - Comparative whole-body PET CT images of the patient at various stages of treatment and post allogenic stem cell transplant. In $\mathbf{A}$ image the inset view shows bilateral breast tumour indicated by two white arrows and a lytic lesion of the rib indicated by yellow arrow

Conflicts of Interest: None of the authors have any conflicts of interest to report in relation to the case work submitted

Data Sharing: Not applicable 

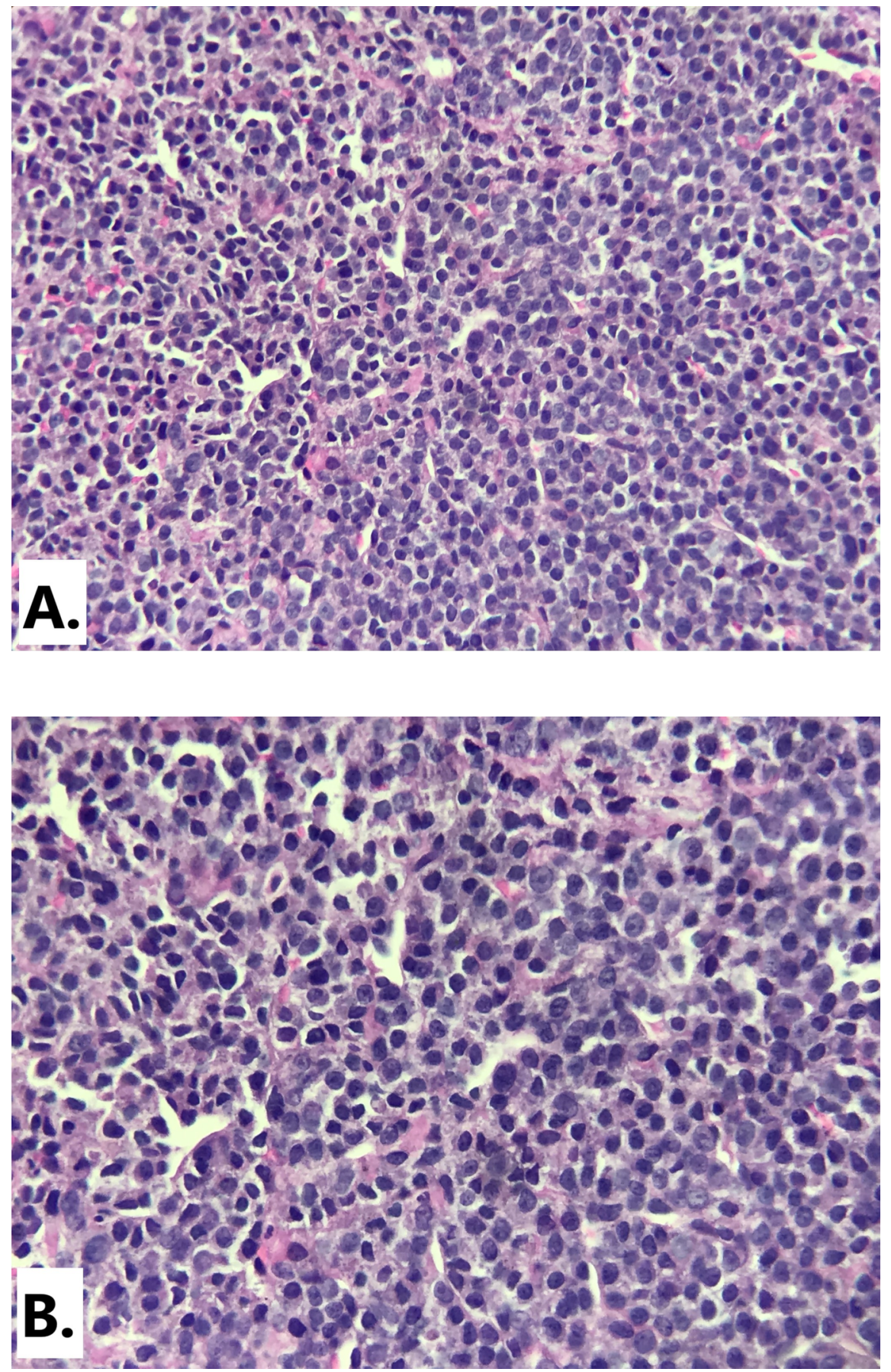

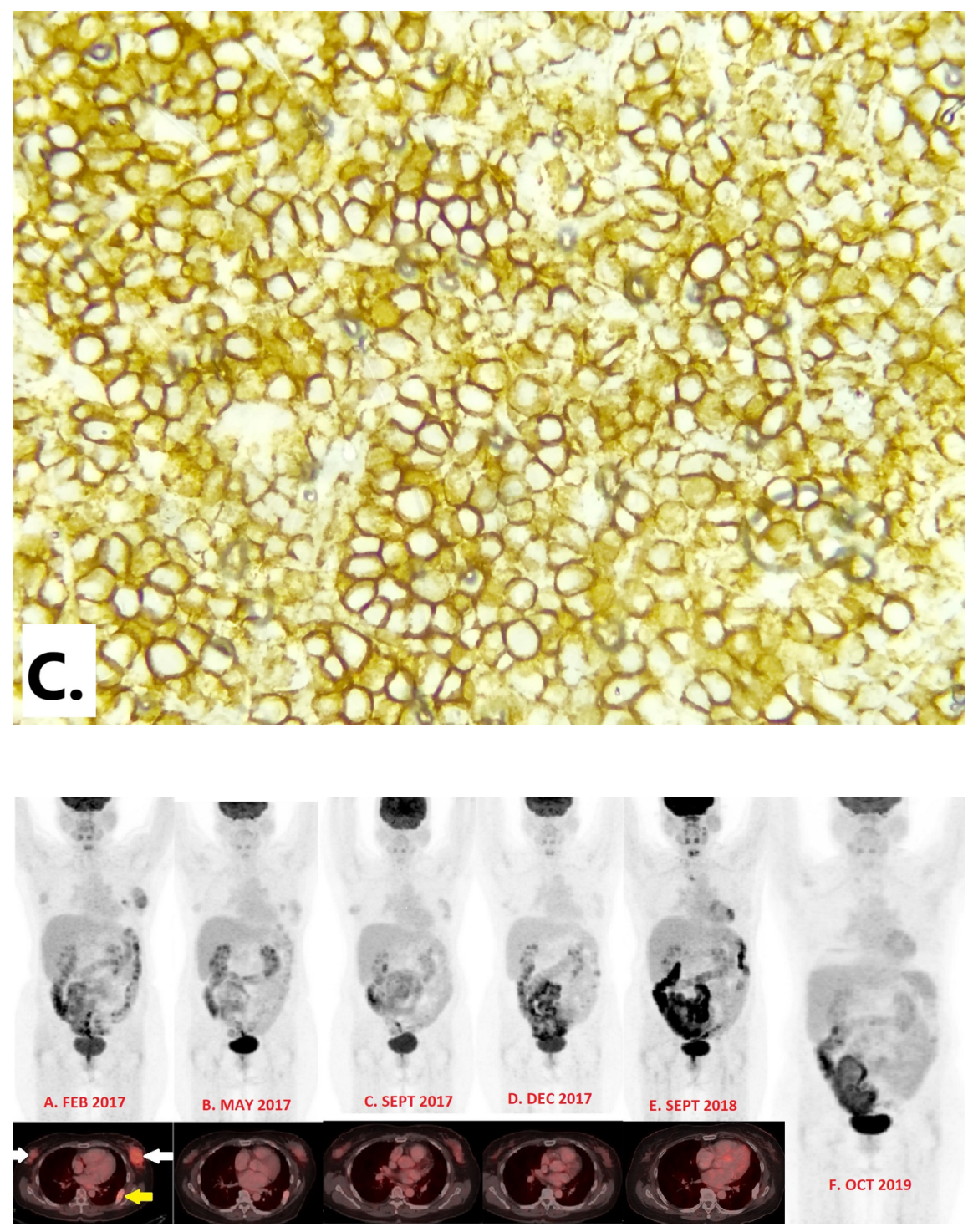